States, Australia and Canada that supply lowcost drugs to the world.

Some countries whose governments purchase drugs with a set budget are also alarmed by signs that the TPP may grant new negotiating powers to the industry. In New Zealand, for example, a government agency called Pharmac determines whether the benefits of a new drug warrant the cost, or if the country is better off sticking with a cheaper alternative. A leaked TPP provision would empower drug companies to appeal such decisions. "We have good processes for ensuring what is for the good of our population, not for the good of lobby groups, and I don't see why they need to interfere with that," says Marilyn Head, a policy analyst at the New Zealand Nurses Organisation in Wellington, who adds: "Bugger off, quite frankly." -

\title{
Electro-optic dye triggers ethics row
}

\section{Dispute puts focus on reporting standards for major grants.}

\section{BY EUGENIE SAMUEL REICH}

$\mathrm{W}$ hen a colleague questions a researcher's hypothesis, how far must the researcher go in telling his prospective funders about those doubts?

The question sits at the heart of a dispute that has prompted a government review of alleged omissions in reports from a science and technology centre funded by grants totalling US\$36 million over 10 years from the National Science Foundation (NSF). The review, by the NSF's inspector general, is not yet complete, but the affair highlights a grey area in the agency's rules for grant recipients: although the rules require principal investigators to disclose any problems they encounter in pursuit of their research goals, they offer no guidance on how to assess when a colleague's scepticism about a specific issue merits reporting.

The issue became public in late July, when Bart Kahr, a chemist at New York University in New York city, described his side of the dispute at a meeting of the American Crystallographic Association in Boston, Massachusetts. But it goes back more than a decade, to work led by Larry Dalton at the University of Washington in Seattle in 2000 . Motivated by the rapid expansion of the Internet, the group was developing modulators, colloquially called 'opto-chips', that convert electrical to optical signals, a more efficient medium for long-distance communication. Dalton and his team reported ${ }^{1}$ recordbreaking performances by electro-optic devices based on dye molecules they had designed. And their paper suggested that the key to the devices' performance lay in the way the molecules lined up in an electric field.

The result was discussed in a 2001 grant proposal to the NSF, which subsequently funded the Center on Materials and Devices for Information Technology Research at the University of Washington, with Dalton as its director. Research continued on the devices, and Kahr joined the centre in 2003. Several groups at the centre and elsewhere were continuing to report improved performances for the devices, but Kahr began to doubt the mechanism that had been proposed to explain how they worked.

Kahr obtained samples of dye molecules from another researcher at the centre, Alex Jen, and measured their absorption of polarized light — a way to test their alignment — in an electric field. Kahr reported to Jen that his results suggested there was no strong alignment and that future efforts to improve the devices by optimizing the dye alignment might not work unless the mechanism was understood. But the centre's annual report to the NSF for 2003-04 did not mention Kahr's findings. Jen, who wrote the relevant section, explains that he had a wealth of material to include, and that there was no effort to omit Kahr's results because they challenged an aspect of the centre's research direction.

Alarmed at what he regarded as an unethical omission, Kahr complained in 2004 to chemist Alvin Kwiram, then the centre's executive director. Kwiram says that Kahr's doubts were a distraction from the centre's main goal, which was to build and improve working devices. Although Kahr believed that understanding the mechanism was necessary to improve the devices as quickly as possible, Kwiram and others felt that they were already being made more effective even though the mechanism was in dispute. "This issue [of the mechanism] was like a mosquito buzzing around and it was like don't bite me right now when we've got bigger fish to fry," Kwiram says.

The centre submitted two more annual reports without mentioning Kahr's finding that the alignment was weak, and in 2006 the centre's grant came up for a five-year renewal. Phil Reid, a chemist at the centre who is now its director, says that during a site visit by NSF reviewers, Jen mentioned theoretical work suggesting that the dye molecules might not be aligned as strongly as supposed - work also mentioned in the 2005-06 annual report

although not in connection with Kahr and his concerns. Kahr says that he did not have an opportunity to present his data to the NSF reviewers, and that he subsequently lost funding he had been receiving through the centre.

Kahr moved to New York University in 2009. In 2011, Reid, Jen, Dalton and Bruce Robinson, a theoretical chemist at the University of Washington, published a paper ${ }^{2}$ presenting their own evidence that some dye molecules similar to those used in the original work align only weakly in an electric field - findings that paralleled those of Kahr. Robinson sees this simply as the resolution of a scientific disagreement, not a matter of research ethics. "Bart was right," says Robinson, "but so what?"

After receiving copies of Kahr's e-mails to centre members raising ethical concerns about the omissions, the University of Washington's Office of Scholarly Integrity and Ana Mari Cauce, dean of the university's College of Arts and Sciences at the time, conducted separate investigations of his allegations in 2010 and 2011. Both cleared Dalton and Jen - the only targets of Kahr's accusations - of

NSF rules offer no guidance on how to assess when a colleague's scepticism merits reporting. any violation of ethics. Cauce, who is now the university's provost, explained in a letter to $\mathrm{Kahr}$ that Jen's omission of Kahr's data from the annual reports was justified because the data were preliminary and because there was a scientific disagreement about whether the molecules were aligned.

But Kahr remained unsatisfied and in January 2011 submitted allegations to the NSF's Office of Inspector General. Susan Carnohan, a spokeswoman for the inspector general, told Nature that the office does not comment on ongoing investigations.

Jason Borenstein, a philosopher who teaches responsible conduct of research to science and engineering students at Georgia Institute of Technology in Atlanta, believes that grant applicants should generally disclose a colleague's doubts in their reports to funders. "Typically it is preferred, if there is space, to say there is another viewpoint that could be presented but we believe ours is right for the following reasons," he says. "That will make a better case to the grant reviewers." -

1. Shi, Y. et al. Science 288, 119-122 (2000).

2. Olbricht, B. C. et al. J. Phys. Chem. B 115, 231-241 (2011). 